\title{
Integrated Design of Dual-Band Antenna with Uni- /Omni-Directional Radiations
}

\author{
Chunxu Mao, Mohsen Khalily, and Pei Xiao \\ Institute for Communication Systems (ICS), Home of the 5G Innovation Centre (5GIC) \\ University of Surrey Guildford, GU2 7XH, UK \\ \{c.mao, m.khalily, p.xiao\}@ surrey.ac.uk
}

\begin{abstract}
A multifunctional antenna with diverse radiation patterns in different frequency bands $(2.45 / 5.8 \mathrm{GHz})$ is presented in this paper. The antenna has a low profile but exhibits an omni-directional radiation pattern in the low-band operation and uni-directional pattern in the high-band operation. For the high-band operation, a $2 \times 2$ patch arrays are designed by employing a out-of-phase feeding method. The low-band operation with the omni-directional pattern is achieved by exciting four open-ended slots in-phase. The four slots are slit in the ground of the high-band array and in this way, this footprint of the antenna is maintained. The operating principles of the antenna are studied with the aid of equivalent circuit model and the current distribution. The antenna is prototyped and measured, demonstrating good results in terms of bandwidths, inter-channel isolation, radiation characteristics.
\end{abstract}

Index Terms-antenna, dual-band, dual-mode, unidirectional, omni- directional.

\section{INTRODUCTION}

Multi-functional antennas with diverse far-field radiation patterns have many potential applications in Internet-ofThings (IoT) and Intelligent Transportation Systems (ITS). Antennas with omni-directional radiation are very useful in many wireless applications where the real-time information exchange between surrounding nodes is desired. On the other hand, antennas with high gain are required to realize the communication with a base station or a satellite. Moreover, a compact size of radio frequency (RF) frontends is highly desired, especially for a multiple antenna system [1]-[3]. To reduce the footprint of a RF frontend, different antennas with different operating modes in the same/different bands should be highly integrated. Besides, high isolation between different antennas is required.

Different radiation modes achieved by using a single structure were reported. In [4]-[7], dual-mode antennas with omni-/uni-directional radiation patterns in two frequency bands were reported. The antennas in [6] and [7] possess linear polarization in the low-band while circular polarization in the high-band. Another feasible method to design the dual-mode antenna is to use two independent antennas. One of the benefits is that different radiating modes can be designed individually, leading to a high flexibility in designing and controlling of the antennas. In

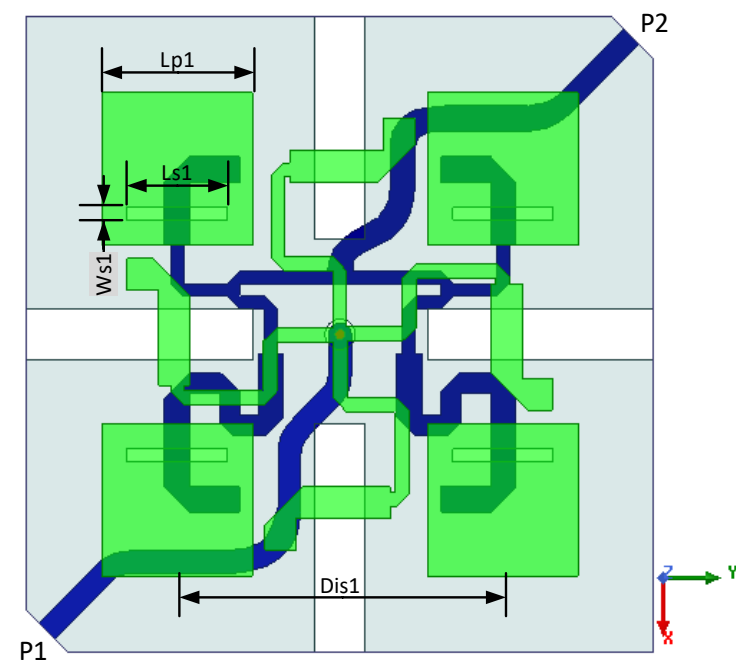

(a)

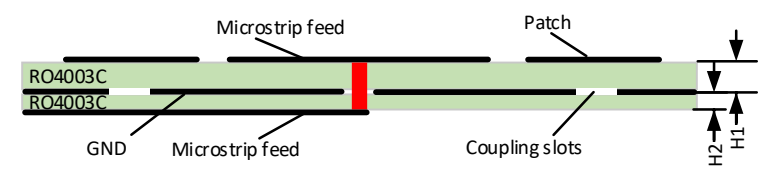

(b)

Fig. 1. Configuration of the proposed dual-band dual-mode antenna (a) top view, (b) side view. $L p l=12 \mathrm{~mm}, L s 1=8 \mathrm{~mm}, W s 1=1 \mathrm{~mm}$, Dis $1=26 \mathrm{~mm}, H 1=1.525 \mathrm{~mm}, H 2=0.8 \mathrm{~mm}$.

[8] and [9], two dual-mode antennas with low profile were proposed in different bands. Even though these reported dual-mode antennas have a compact size, they suffer from limited bandwidths and performance degradation when the two modes are excited simultaneously.

In this paper, a highly integrated antenna with omni- and uni-directional radiation patterns in two bands is proposed. The antenna has the omni-directional pattern in the lowband operation but broadside pattern in the high-band. To reduce the size, the dual-mode operation is achieved through an integrated design that the low-band and high-band operations are fully combined. To achieve the omnidirectional radiation pattern, a novel method is proposed by exciting four rotated open-ended slots in the ground of the high-band operation. Therefore, a high isolation between the two modes can be maintained in a compact footprint. 


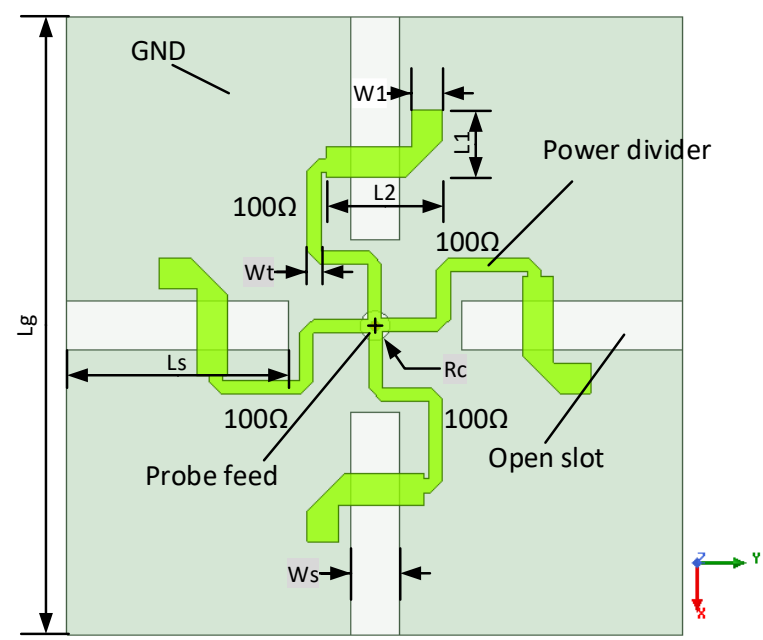

Fig. 2. Configuration of the low-band omni-directional antenna: $L g=40$ $\mathrm{mm}, L s=18 \mathrm{~mm}, W s=4 \mathrm{~mm}, L 1=4.5 \mathrm{~mm}, L 2=9.5 \mathrm{~mm}, W 1=2.5$ $\mathrm{mm}, W t=0.8 \mathrm{~mm}, R c=1.2 \mathrm{~mm}$.

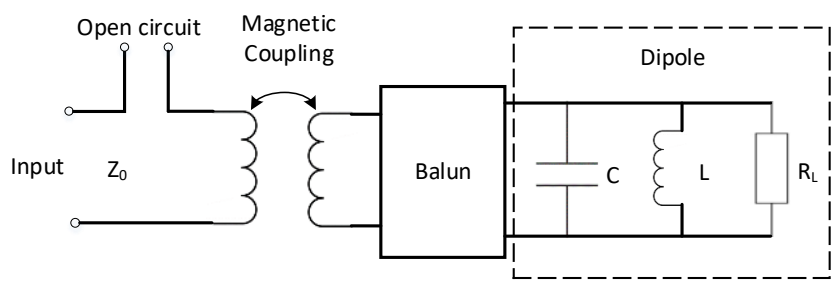

Fig. 3. Equivalent circuit of the microstrip-fed slot.

\section{ANTENNA DESIGN}

\section{A. Antenna Configurations}

Figure 1(a) shows the configuration of the proposed dualband dual-mode antenna. The antenna is comprised of a $2 \times$ 2 linearly polarized (LP) microstrip patch array for the highband operation and four rotated slots etched in the ground plane. The high-band patch array is fed by a microstrip power splitting network via the four slots in the ground plane. The low-band antenna is achieved by simultaneously exciting the four open-ended slots through a four-way power splitter, which is printed on the top layer of the upper substrate, sharing the same layer with the patch array. The microstrip feeding networks are printed on the bottom layer of the lower substrate. The ground plane in the middle not only functions as the ground plane of the patch array but also the radiating element of the low-band antenna. Fig. 1(b) shows the stacked configuration of the antenna. The lowband power splitter on the top layer is connected to the microstrip line via a metallic hole. Rogers RO4003C with a relative permittivity of 3.55 and a loss tangent of 0.0027 is used as a substrate in the design. Optimization of the antenna performance was performed using the High Frequency Structural Simulator (HFSS) and the optimized parameters are listed in the caption of Fig. 1.

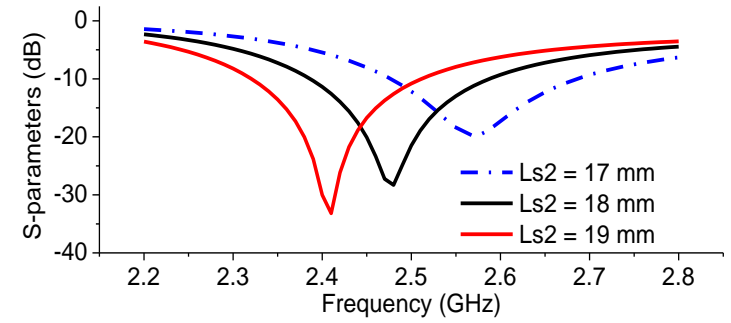

(a)

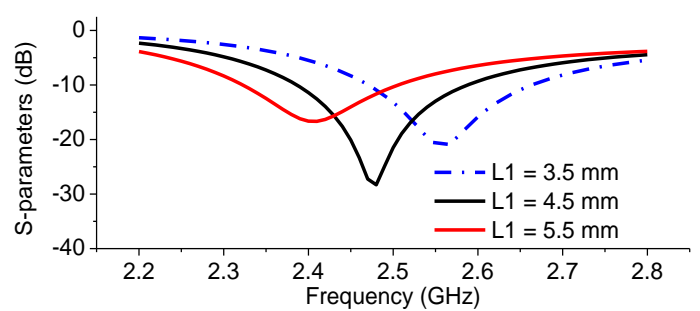

(b)

Fig. 4. Simulated $S_{11}$ of the low-band antenna with different (a) lengths of the open slot, $L s$ (b) lengths of the feed line, $L 1$.

\section{B. Low-Band Antenna and Omni-Directional Radiation Implementation}

To better illustrate the implementation of the low-band antenna, the patches and corresponding feeding networks of the high-band operation shown in Fig. 1 are removed, as shown in Fig. 2. The low-band antenna consists of four open-ended slots in the ground plane fed by a 4-way power splitting network. The four arms of the power splitter have a symmetrical structure, ensuring that the four slots can be excited in-phase. The final optimized geometrical parameters are listed in the caption of Fig. 2.

Figure 3 illustrates the equivalent circuit of the slot antenna element. The radiator is the two metallic edges on both sides of the slot which can be equivalent as a shunt RLC resonator. The slot is served as a balun, which is used to balance the input power on the conductive plane. The power is coupled to the slot from the microstrip lines. It is noted that the open ended microstrip feed line can also be used to tune the impedance matching performance. In this work, the resonant frequency of the antenna is dominated by the radiating section, i.e. the size of the ground plane, $L g$. The dimensions of the slot can also be used to adjust the resonant frequency of the antenna. Fig. 4 shows the simulated S-parameters of the low-band antenna with different lengths of the open-ended slot, Ls, and the microstrip feed line, L1. As observed, the resonant frequency shifts to the lower band as $L s$ and $L 1$ are increased. This provides two additional methods to control the resonant frequency of the antenna without changing the overall size of the ground plane. In this work, $L s=18 \mathrm{~mm}$ and $L 1=4.5 \mathrm{~mm}$ were chosen.

The principles of achieving the omni-directional radiation pattern in xy-plane can be explained by studying the current distribution at $2.45 \mathrm{GHz}$, as depicted in Fig. 5(a). As it can be observed, when the four open-ended slots are excited inphase, producing four current paths along the edges of the 


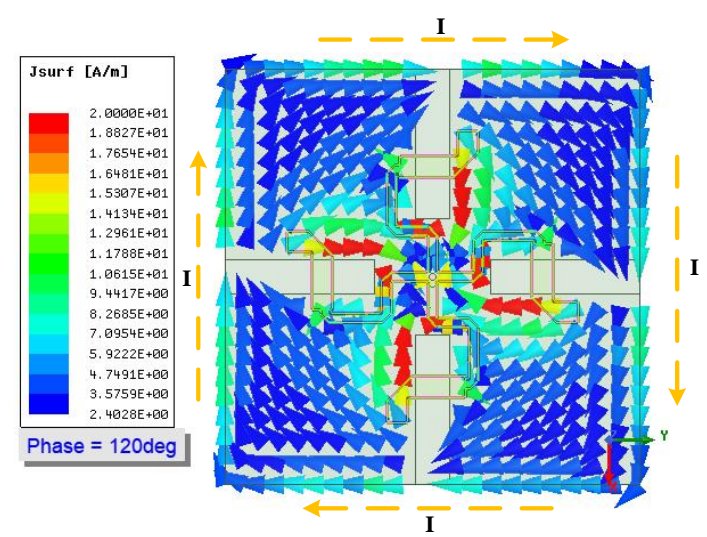

(a)
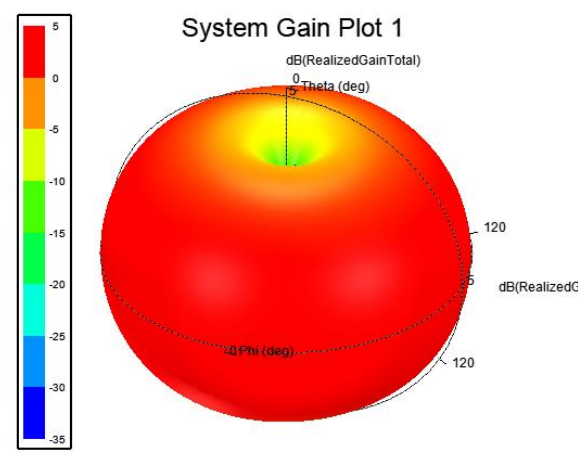

(b)

Fig. 5. (a) Simulated current distribution of the rotation-fed antenna at $2.45 \mathrm{GHz}$ and (b) simulated 3-D radiation pattern at 2.45.

ground plane. The four current flows are combined to form a magnetic-dipole-like current distribution along the edge of ground plane, which generates an omni-directional radiation pattern in the far-field. Fig. 5(b) shows the simulated 3-D radiation pattern at $2.45 \mathrm{GHz}$. Good omni-directional radiation in the xy-plane is realized.

\section{Inter-Channel Isolation}

For a dual-mode antenna, it is essential to have a high isolation between the two operating modes. To illustrate it, the simulated current distributions at 2.45 and $5.85 \mathrm{GHz}$ are shown in Fig. 6. As seen, when the antenna works at 2.45 $\mathrm{GHz}$, the current is mainly flowing along the slots and contour of the ground plane. In contrast, when port 2 is excited at $5.85 \mathrm{GHz}$, the current is mainly distributed in the central area while the current strength around the open-ended slots and ground edges is very week. The different current distributions show that the two operating modes of the antenna can function independently without incurring much interference. This is due to different sections of the antenna are used for different operating modes, and high isolation between the two modes can be achieved even they are closely placed together.

\section{RESULTS AND DISCUSSION}

Figure 7 shows each layer of the antenna prototype. The measured S-parameters of the antenna are compared with

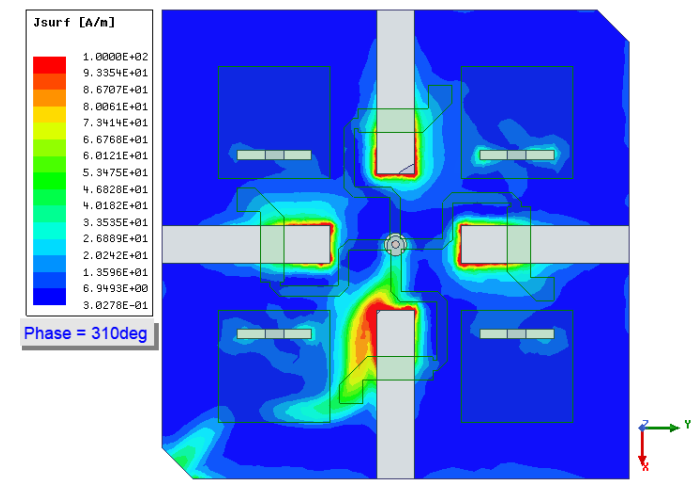

(a)

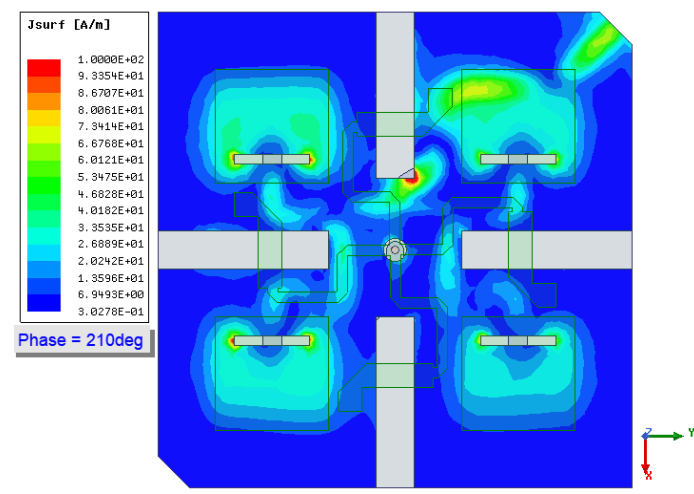

(b)

Fig. 6. Simulated current distribution of the proposed dual-mode antenna: (a) $2.45 \mathrm{GHz}$, port 1 is excited, (b) $5.85 \mathrm{GHz}$, port 2 is excited.

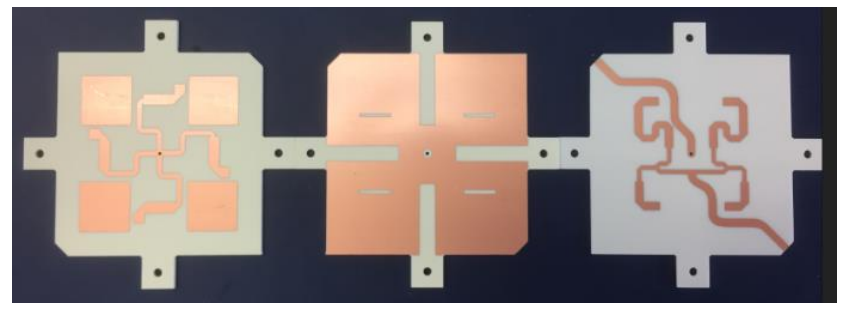

(a)

(b)

(c)

Fig. 7. Prototype of the proposed dual-band dual-mode antenna: (a) top layer, (b) middle layer, (c) bottom layer.

the simulation results in Fig. 8. The measured results agree very well with the simulations at both bands, showing an impedance bandwidth from 2.38 to $2.56 \mathrm{GHz}$ for the lowband operation (port-1 is excited) and a high-band ranging from 5.7 to $6.15 \mathrm{GHz}$ for the high-band operation (port-2 is excited). Moreover, a high isolation of $22 \mathrm{~dB}$ is achieved between the two operation modes. The minor discrepancy is attributed to fabrication and assembly tolerances.

Figure 9 shows the simulated and measured gains of the proposed dual-mode antenna at both bands. When port-1 is excited in the low band, the antenna exhibits an omnidirectional radiation pattern with a gain of $2 \mathrm{dBi}$. When port- 2 is excited in the high-band, a high gain of over $10 \mathrm{dBi}$ is achieved due to the antenna has a uni-directional radiation. The measured results agree well with the simulations in both two frequency bands. 


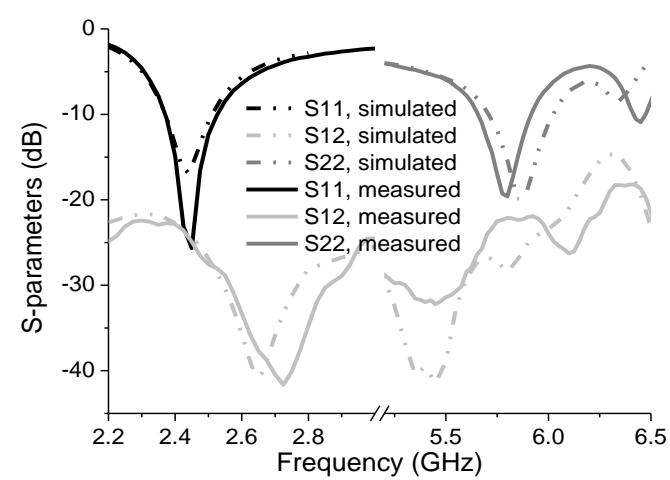

Fig. 8. Simulated and measured S-parameters of the proposed dualmode antenna.

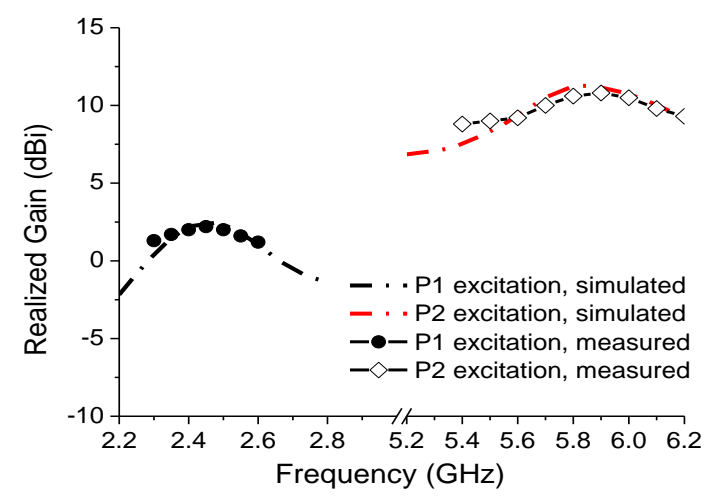

Fig. 9. Simulated and measured realized gains of the proposed dualmode antenna when the two ports are excited, respectively.

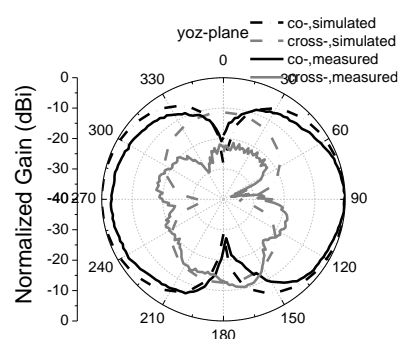

(a)

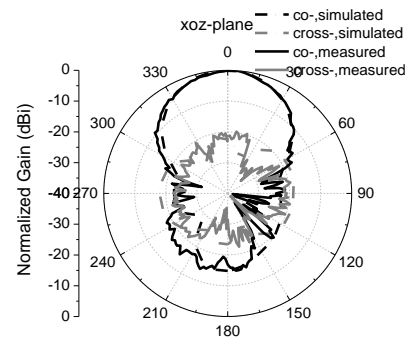

(c)

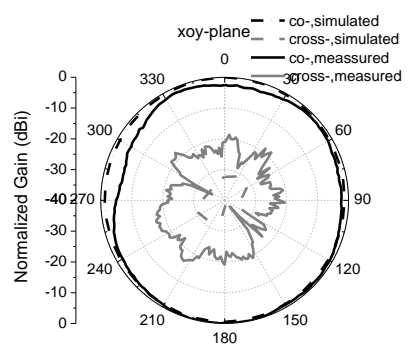

(b)

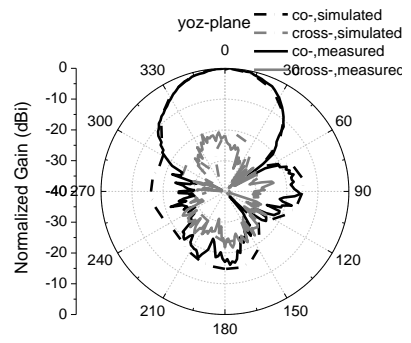

(d)
Fig. 10. Simulated and measured normalized radiation patterns: (a) P1 is excited, $2.45 \mathrm{GHz}$, xz-plane, (b) P1 is excited, $2.45 \mathrm{GHz}$, xy-plane, (c) P2 is excited, $5.85 \mathrm{GHz}$, xz-plane, (d) P2 is excited, $5.85 \mathrm{GHz}$, yz-plane.

Figure 10 shows the simulated and measured normalized radiation patterns of the dual-mode antenna when the two ports are excited, respectively. It is observed from Fig. 10(a) and (b) that the antenna has a dumbbell-like radiation pattern in xz-plane and an omni-directional radiation pattern in xy-plane when the port-1 is excited in the low-band. It should be noted that the electric-field plane of the antenna is in the horizontal plane when it operates in its omnidirectional mode. The measured cross-polarization discrimination (XPD) is around $20 \mathrm{~dB}$, which is slightly lower than the simulation. When port- 2 is excited, in contrast, the antenna exhibits the uni-directional radiation in the broadside direction with linear polarization along the $\mathrm{x}$ axis direction, as shown in Fig. 10(c)-(d). The front-to-back ratio is over $16 \mathrm{~dB}$. Moreover, a good XPD of over $20 \mathrm{~dB}$ is achieved in both orthogonal planes due to the adoption of out-of-phase feeding networks. Finally, the measured results agree reasonably well with the simulations.

\section{CONCLUSION}

A compact, highly integrated dual-band dual-mode antenna with omni-/uni-directional radiation characteristics is proposed. The antenna has an omni-directional radiation patterns in the low-band for the first mode operation and a broadside radiation pattern in the high-band for the second mode operation. Due to a novel integration design is adopted, the antenna has a compact size and low profile but a high degree of isolation between the two modes. The operating principles of realizing omni-directional radiation in the low-band were studied. The proposed dual-mode antenna can be a good candidate for potential IoT and ITS applications.

\section{REFERENCES}

[1] J. Tak, S. Woo, J. Kwon, and J. Choi, "Dual-band dual-mode patch antenna for on-/off-body WBAN communications," IEEE Antennas Wireless Propag. Lett., vol. 15, pp. 348-351, 2016.

[2] D. H. Werner and Z. H. Jiang, Electromagnetics of Body Area Networks: Antennas, Propagation, and RF Systems, Wiley-IEEE Press, 2016.

[3] C. Mao, S. Gao, Y. Wang, "Dual-band full-duplex Tx/Rx antennas for vehicular communications", IEEE Trans. Veh. Technol., vol. 67, no. 5, pp. 4059-4060, May 2018.

[4] X. Y. Zhang, H. Wong, T. Mo, Y. F. Cao, "Dual-band dual-mode button antenna for on-body and off-body communications," IEEE Trans. Biomed. Circuits Syst., vol. 11, no. 4, pp. 933-941, Apr. 2017.

[5] D. Sarkar, K. V. Srivastava, "Comparct dual-band dual-mode microstrip-fed dipole-loop antennas for pattern diversity arrays," Electron. Lett., vol. 53, no. 10, pp. 639-640, May 2017.

[6] J. Lin, Z. Qian W. Cao, S. Shi, Q. Wang, W. Zhong, "A low-profile dual-band dual-mode and dual polarized antenna based on AMC," IEEE Antennas Wireless Propag. Lett., vol. 16, pp. 2473-2476, 2017.

[7] W. Q. Cao, "Compact dual-band dual-mode circular patch antenna with broadband unidirectional linearly polarised and omnidirectional circularly polarized characteristics," IET Microw. Antennas Propag., vol. 10, no. 2, pp. 223-229, Apr. 2016.

[8] Y. Hong, J. Tak and J. Choi, "Dual-band dual-mode patch antenna for on-off WBAN applications," Electron. Lett., vol. 50, no. 25, pp. 1895-1896, 2014.

[9] R. Masood, C. Person, R. Sauleau, "A dual-mode dual-port pattern diversity antenna for $2.45-\mathrm{GHz}$ WBAN," IEEE Antennas Wireless Propag. Lett., vol. 16, pp. 1064-1067, 2017. 